\begin{tabular}{|c|l|}
\hline Title & Local invariants of singular surfaces in an al most complex four-manifold \\
\hline Author(s) & Ishikawa, Goo; Ohmoto, Toru \\
\hline Citation & Hokkaido University Preprint Series in Mathematics, 143, 2-9 \\
\hline Issue Date & 1992-03 \\
\hline DOI & 10.14943/83287 \\
\hline Doc URL & http://hdl.handle.net/2115/68889 \\
\hline Type & bulletin (article) \\
\hline File Information & pre143.pdf \\
\hline
\end{tabular}

Instructions for use 
Local invariants of singular surfaces in an almost complex four-manifold

Goo Ishikawa and Toru Ohmoto

Series $\sharp 143$. March 1992 


\section{HOKKAIDO UNIVERSITY \\ PREPRINT SERIES IN MATHEMATICS}

$\sharp 117$ : T. Hibi, A lower bound theorem for Ehrhart polynomials of convex polytopes, 6 pages. 1991 .

$\sharp 118$ : R. Agemi, H. Takamura, The lifespan of classical solutions to nonlinear wave equations in two space dimensions, 30 pages. 1991.

$\sharp 119$ : S. Altschuler, S. Angenent and Y. Giga, Generalized motion by mean curvature for surfaces of rotation, 15 pages. 1991.

\#120: T. Nakazi, Invariant subspaces in the bidisc and commutators, 20 pages. 1991.

$\sharp 121$ : A. Arai, Commutation properties of the partial isometries associated with anticommuting self-adjoint operators, 25 pages. 1991.

$\sharp 122: \quad$ Y.-G. Chen, Blow-up solutions to a finite difference analogue of $u_{t}=\Delta u+u^{1+\alpha}$ in $N$-dimensional balls, 31 pages. 1991.

$\sharp 123: \quad$ A. Arai, Fock-space representations of the relativistic supersymmetry algebra in the two-dimensional spacetime, 13 pages. 1991.

$\sharp 124: \quad$ S. Izumiya, The theory of Legendrian unfoldings and first order differential equations, 16 pages. 1991.

$\sharp 125: \quad$ T. Hibi, Face number inequalities for matroid complexes and Cohen-Macaulay types of Stanley-Reisner rings of distributive lattices, 17 pages. 1991.

\#126: S. Izumiya, Completely integrable holonomic systems of first order differential equations, 35 pages. 1991.

$\sharp 127$ : G. Ishikawa, S. Izumiya and K. Watanabe, Vector fields near a generic submanifold, 9 pages. 1991.

$\sharp 128$ : A. Arai, I. Mitoma, Comparison and nuclearity of spaces of differential forms on topological vector spaces, 27 pages. 1991.

$\sharp 129$ : K. Kubota, Existence of a global solution to a semi-linear wave equation with initial data of non-compact support in low space dimensions, 53 pages. 1991.

$\sharp 130: \quad$ S. Altschuler, S. Angenent and Y. Giga, Mean curvature flow through singularities for surfaces of rotation, 62 pages. 1991.

$\sharp 131$ : M. Giga, Y. Giga and H. Sohr, $L^{p}$ estimates for the Stokes system, 13 pages. 1991.

$\sharp 132$ : Y. Okabe, T. Ootsuka, Applications of the theory of $\mathrm{KM}_{2} \mathrm{O}$-Langevin equations to the non-linear prediction problem for the one-dimensional strictly stationary time series, 27 pages. 1992.

$\sharp 133$ : Y. Okabe, Applications of the theory of $\mathrm{KM}_{2} \mathrm{O}$-Langevin equations to the linear prediction problem for the multi-dimensional weakly stationary time series, 22 pages. 1992.

\#134: P. Aviles, Y. Giga and N. Komuro, Duality formulas and variational integrals, 22 pages. 1992.

$\sharp 135$ : S. Izumiya, The Clairaut type equation, 6 pages. 1992.

\# 136: S. Izumiya, Singular solutions of first order differential equations, 6 pages. 1992.

$\sharp 137$ : S. Izumiya, W.L. Marar, The Euler characteristic of a generic wave front in a 3-manifold, 6 pages. 1992.

$\$ 138$ : S. Izumiya, W.L. Marar, The Euler characteristic of the image of a stable mapping from a closed n-manifold to a $(2 n-1)$-manifold, 5 pages. 1992.

$\sharp 139$ : Y. Giga, Z. Yoshida, A bound for the pressure integral in a plasma equilibrium, 20 pages. 1992.

$\sharp 140: \quad$ S. Izumiya, What is the Clairaut equation ?, 13 pages. 1992.

$\sharp 141: \quad$ H. Takamura, Weighted deformation theorem for normal currents, 27 pages. 1992.

$\sharp 142$ : T. Morimoto, Geometric structures on filtered manifolds, 104 pages. 1992. 


\title{
Local invariants of singular surfaces in an almost complex four-manifold
}

\section{GOO ISHIKAWA ${ }^{\dagger}$ AND TORU OHMOTO OH $^{\ddagger}$}

\author{
$\dagger$ Department of Mathematics, Hokkaido University, Sapporo 060, Japan, \\ $\ddagger$ Department of Mathematics, Tokyo Institute of Technology, Ohokayama 152, Japan, \\ and Department of Mathematics, Hokkaido University, Sapporo 060, Japan.
}

\section{Introduction.}

Let $\left(M^{4}, J\right)$ be an almost complex manifold of dimension 4, $S$ an oriented closed surface, and $f: S \rightarrow(M, J)$ a $C^{\infty}$ mapping. Then $f$ has two sorts of singularities: "complex points" and "non-immersive points". As well as these singularities, they appear also multi-singular points.

Associated to these singularities, we define in this paper two local invariants : the local self-intersection index $i$ at a point of $f(S)$ and the Maslov index $m$ at a point of $S$, for a generic $f$ belonging to the complement of an infinite codimensional subset in the space $C^{\infty}(S, M)$ of $C^{\infty}$ mappings from $S$ to $M$ endowed with the $C^{\infty}$ topology. (See $\S 1$ for the precise definitions of the genericity and the invariants.)

Immersed surfaces in a four-space are studied by many authors from various aspects (e.g. [6],[5],[17],[11],[8],[9],[2], see also the reference of [4]). Also remark that similar local invariants as $i$ and $m$ are already defined and investigated in the contrary case, that is, for (pseudo-)holomorphic curves, ([18], [10]).

Then we show the following formulae

ThEOREM 1. Let $f: S \rightarrow(M, J)$ be generic. Set $V=f(S)$. Then

$$
\text { (1) } \sum_{y \in V}\{i(y)+1\}=\chi(V)+V \cdot V, \quad \text { (2) } \quad \sum_{x \in S} m(x)=c \text {. }
$$

Here $\chi(V), V \cdot V$ and $c$ are global numerical invariants; $\chi(V)$ is the Euler characteristic of $V, V \cdot V$ is the self-intersection index of $V$ in $M$ and $c=\left\langle c_{1}\left(f^{*} T M\right),[S]\right\rangle$ is the Chern number. Remark that $M$ has the natural orientation from the almost complex structure $J$. Since we see below $i(y)=-1$ and $m(x)=0$ except finite points, the left hand side of each formula has a meaning.

Theorem 1 turns out to unify and generalize two sorts of known formulae.

For an immersion $f$ of $S$ into $M$, it is known the following formula due to Lai [17],(see also [3],[4],[2]): If the complex points of $f$ are all transverse, then

$$
d_{+}+d_{-}=\chi+\nu, \quad d_{+}-d_{-}=c .
$$


Here $\chi$ is the Euler characteristic of $S$ and $\nu$ is the normal Euler number of $f$, whereas $d_{ \pm}=e_{ \pm}-h_{ \pm}$with

$e_{+}=\sharp$ (positive elliptic point), $e_{-}=\sharp$ (negative elliptic point),

$h_{+}=\sharp$ (positive hyperbolic point), $h_{-}=\sharp$ (negative hyperbolic point).

See $\S 1$ for the notions.

The immersion $f$ can be approximated so that $V=f(S)$ has only transverse selfintersections on non-complex points. Then the invariants appeared in Lai's formula do not vary and Theorem 1 implies Lai's formula in a simple manner, if we calculate $i$ and $m$ for some special singular points.

In the symplectic situation, on the other hand, it is known a formula due to Givental' on the self-intersection index of a "Lagrange cycle" ([13],[14],[1]): Let $\left(M^{4}, \omega\right)$ be a symplectic manifold of dimension 4 and $f: S \rightarrow(M, \omega)$ be an isotropic $C^{\infty}$ mapping, $\left(f^{*} \omega=0\right)$. Remark that $M$ has the orientation coming from $\omega^{2}$. If $V=f(S)$ has the open Whitney umbrellas and the transverse self-intersections as singularities, then the formula is

$$
-V \cdot V=\chi-2 \delta+T
$$

Here $\delta$ is the sum of intersection indices of self-intersection points and $T$ is the number of open Whitney umbrellas. An open Whitney umbrella has a local model $f_{2,1}: R^{2}, 0 \rightarrow R^{4}, 0$ defined by

$$
f_{2,1}(u, v)=\left(p_{1}, q_{1}, p_{2}, q_{2}\right)=\left(v^{3} / 3, u, u v, v^{2} / 2\right), \quad \omega=d p_{1} \wedge d q_{1}+d p_{2} \wedge d q_{2},
$$

([16]). (The original form of the formula in [13] is $V \cdot V=\chi+2 \sharp+T, \sharp=\delta$, because the orientation of $M$ chosen in [13] differs by sign with the orientation chosen here.)

We remark that, for a symplectic manifold $(M, \omega)$, there exists an almost complex structure $J$ unique up to homotopy such that $\omega(\cdot, J \cdot)$ is positive definite (see [22]). Then an isotropic immersion has no complex points.

Thus we can apply Theorem 1 to this situation.

Theorem 1 follows also that, if $f^{*} T M$ has a Lagrange subbundle, then $c_{1}\left(f^{*} T M\right)=0$ and therefore the sum of Maslov indices is equal to zero. This fact is first observed also by Givental' [13] in the simplest case.

The formula of Givental' is generalized in some sense to higher dimensional cases as formulae on "isotropic Thom polynomials" [20].

We also remark that, using Viro's integral formulation based on Euler characteristics [21], the formulae of Theorem 1 can be written in the following form:

$$
\text { (1) } \int_{y \in V} i(y) d \chi(y)=V \cdot V, \quad \text { (2) } \int_{y \in V} m(y) d \chi(y)=c \text {, }
$$

where $m(y)=\sum_{x \in f^{-1}(y)} m(x)$. Regarding the Chern number as the global counterpart of the Maslov index, we can observe each formula has a natural form that integrating a local invariant gives a global one. 
The proof of Theorem 1 is simple if once the definitions of $i$ and $m$ are established.

Next we turn the local situation relatively to $S$. Let $f: \mathrm{R}^{2}, 0 \rightarrow(M, J)$ be a generic map-germ. Then two invariants $i(f)$ and $m(f)$ can be defined as $i(f)=i(f(0))$ and $m(f)=m(0)$ respectively.

After taking a representative $f: D^{2} \rightarrow(M, J), D^{2}=\left\{(u, v) \in \mathrm{R}^{2} \mid u^{2}+v^{2}<\epsilon^{2}\right\}$ for a sufficiently small $\epsilon$, we perturb $f$ into an immersion $\tilde{f}$ with transverse self-intersections such that all complex points of $\tilde{f}$ are transverse. Then we have the following formula on perturbations:

THEOREM 2. $i(f)=d_{+}+d_{-}-1+2 \delta, \quad m(f)=d_{+}-d_{-}$. 2.1.)

Notice that the numbers $d_{+}, d_{-}$and $\delta$ depend on a perturbation of $f$. (See Example As a corollary we see $i(f) \equiv m(f)+1, \bmod$. 2 .

Beside the definitions of $i$ and $m$, we need some calculations of them to prove Theorem 2, and also to show that Theorem 1 implies formulae of Lai and Givental' respectively. We gather the results into the following table:

Table 3.

\begin{tabular}{|c|c|c|}
\hline type of the singularity & $i$ & $m$ \\
& & \\
\hline non-singular point & -1 & 0 \\
\hline open Whitney umbrella & -2 & \pm 1 \\
\hline self-intersection of index +1 & 0 & 0 \\
\hline self-intersection of index -1 & -4 & 0 \\
\hline positive elliptic point & 0 & +1 \\
\hline negative elliptic point & 0 & -1 \\
\hline positive hyperbolic point & -2 & -1 \\
\hline negative hyperbolic point & -2 & +1 \\
\hline
\end{tabular}

We can deduce Theorem 1 contrary from Lai's formula, perturbing $f$ and applying Theorem 2 and the results in Table 3. Thus Lai's formula is generalized to the simple formula (Theorem 1), the difficulty being pushed into the calculations of invariants.

In $\S 1$ we define $i$ and $m$. In the next section we prove Theorem 2 . The calculation of $i$ and $m$ (Table 3 ) are given in $\S \S 1$ and 2 . Theorem 1 is proved in $\S 3$.

In this paper manifolds and mappings are assumed of class $C^{\infty}$. 


\section{Genericity and local invariants.}

Let $f: S \rightarrow(M, J)$ be a mapping. An immersive point $x \in S$ of $f$ is called a complex point if $f_{*} T_{x} S=J\left(f_{*} T_{x} S\right)$.

DEFINITION 1.1: $f$ is called generic if (1) $f$ is finite to one and for any $y \in V=f(S)$, (2) the germ $f: S, f^{-1}(y) \rightarrow M, y$ is an embedding with no complex points outside of $f^{-1}(y)$ and (3) the pull-back by $f$ of a positive definite Morse function around $y$ is of finite multiplicity at $f^{-1}(y)$.

A map-germ $f: S, x \rightarrow(M, J)$ is called generic if, for $y=f(x),(2)$ and (3) hold, $f^{-1}(y)$ being replaced by $x$.

REMARK 1.2: Non generic mappings form an infinite codimensional subset in $C^{\infty}(S, M)$, even after more strict restrictions on genericity are imposed (see [12] for instance), since, in the 1-jet space $J^{1}(S, M)$ the totality of immersive 1-jets corresponding to complex points is of codimension 2, (see [17]).

Let $f$ be generic. Then $V$ is a totally real submanifold of $(M, J)$ except for finite points.

Now we intend to define $i(y) \in \mathbb{Z}$ for $y \in V$ as the self-intersection index $V$ at $y$. To do this, we have to assign a perturbation of $V$ near $y$.

For a sufficiently small sphere $S^{3}$ centered $y$ in $M$ (with respect to some coordinate), $f$ is transverse to $S^{3}$ by the property (3) of Definition 1.1. Considering the link $L=V \cap S^{3}$, we take a tangent vector field $v$ to $V$ defined near $L$ and directed outward. Then the field $J v$ does not tangent to $V$ by the property (2). Thus we perturb $f$ into $f^{\prime}$ along the direction $J v$ and count intersection indices of $V$ and $f^{\prime}(S)$ near $y$. In other word, we adopt the following definition:

DEFINITION 1.2: (Local self-intersection index of $f$ at $y$.) We set $i(y)=\operatorname{link}\left(L, L^{\prime}\right)$, where $L^{\prime}=f^{\prime}(S) \cap S^{3}$.

Clearly $i(y)$ does not depend on the choice of $S^{3}$ and $v$.

Since, on an immersed surface without complex points, multiplying $J$ maps the tangent bundle isomorphically to the normal bundle with the reverse orientation, it is easy to verify

LEMMA 1.3. If $y \in V$ is a non-singular point, then $i(y)=-1$. If $y \in V$ is a transverse selfintersection (non-complex) point, then $i(y)=0,-4$, according to the intersection index is $+1,-1$, respectively.

REMARK 1.4: If $J_{t}$ is a homotopy of complex structures such that $J_{0}=J$ and $V$ has no complex points near $L$ with respect to $J_{t}$. Then the number $i(y)$ with respect to $J_{t}$ does not depend on $J_{t}$. Similar result holds also for $m$ defined below.

We next define the Maslov index $m(x)$ for $x \in S$, generalizing the definition in [13].

Consider the $\mathbb{C}^{2}$-bundle $E=f^{*} T M$ over $S$. Let $\tilde{G}$ denote the space of oriented 2planes in $E$, and $\pi: \tilde{G} \rightarrow M$ the canonical projection. The fiber of $\pi$ is $G=G_{4,2}$, the Grassmannian of oriented 2-planes in $\mathbb{C}^{2}$.

Let $\tilde{C} \subset \tilde{G}$ be the totality of complex planes. We decompose $\tilde{C}=\tilde{C}_{+} \cup \tilde{C}_{-}$, where an oriented plane $\alpha \in \tilde{C}$ belongs to $\tilde{C}_{+}$if and only if the orientation of $\alpha$ coincides with the 
orientation as the complex plane. Then we see $\tilde{C}_{ \pm}$are submanifolds of $\tilde{G}$ of codimension 2 respectively.

We define orientations of $\tilde{G}, \tilde{C}_{+}$and $\tilde{C}_{-}$as follows (cf. [17],[9],[3],[4]): For each $x \in S$, we take a local frame $e_{1}, e_{2}, e_{3}, e_{4}$ of $E$ as $\mathrm{R}^{4}$-bundle near $x$ with $J e_{1}=e_{2}, J e_{3}=e_{4}$. Then we set

$$
\left\{\begin{array} { l } 
{ x _ { 1 } = p _ { 1 2 } + p _ { 3 4 } , } \\
{ x _ { 2 } = p _ { 2 3 } + p _ { 1 4 } , } \\
{ x _ { 3 } = p _ { 3 1 } + p _ { 2 4 } , }
\end{array} \quad \left\{\begin{array}{l}
y_{1}=p_{12}-p_{34}, \\
y_{2}=p_{23}-p_{14}, \\
y_{3}=p_{31}-p_{24},
\end{array}\right.\right.
$$

for the Plücker coordinate $p_{i j}$. Then we identify $G$ with $\left(\mathbf{R}^{3}-0\right) / \mathbf{R}_{>0} \times\left(\mathbf{R}^{3}-0\right) / \mathbf{R}_{>0} \cong$ $S^{2} \times S^{2}$ by these coordinates. The fiber of $\tilde{C}_{+}$(resp. $\tilde{C}_{-}$) corresponds to $C_{+}=n \times S^{2}$ (resp. $C_{-}=s \times S^{2}$ ), where $n=(1,0,0), s=(-1,0,0)$.

We orient $\tilde{G}$ (resp. $\tilde{C}_{+}, \tilde{C}_{-}$) from the orientations of $S$ and $G$ (resp. $C_{+}, C_{-}$). We denote by $-\tilde{C}_{-}$the $\tilde{C}_{-}$with the reverse orientation.

Let $\Sigma \subset S$ be the set of non-immersive points of $f$. Then we define the Gauss mapping $g: S-\Sigma \rightarrow \tilde{G}$ by $g(x)=f_{*}\left(T_{x} S\right), x \in S-\Sigma$.

For $x \in S$, we take a small loop $\ell$ around $x$. Then $g \circ \ell$ extends to a section $\tilde{g}$ over the disk, since $\tilde{G}$ is a $S^{2} \times S^{2}$-bundle. We count the intersection number of $\tilde{g}$ with $\tilde{C}_{+} \cup\left(-\tilde{C}_{-}\right)$. In other word, we adopt the following definition:

Definition 1.5: (The Maslov index of $f$ at $x$.) We set $m(x)=\operatorname{link}\left(g \circ \ell, \tilde{C}_{+} \cup\left(-\tilde{C}_{-}\right)\right.$).

If $x \in S$ is a complex point, then $g(x) \in \tilde{C}$.

Definition 1.6: A complex point $x \in S$ is called positive (resp. negative) if $g(x) \in \tilde{C}_{+}$ (resp. $g(x) \in \tilde{C}_{-}$). A complex point $x \in S$ is called transverse if $g$ is transverse to $\tilde{C}$ at $x$. A transverse complex point $x$ is elliptic (resp. hyperbolic) if the intersection index of $g$ and $\tilde{C}=\tilde{C}_{+} \cup \tilde{C}_{-}$at $g(x)$ is equal to +1 (resp. -1 ).

Then the following is straightforward.

LEMMA 1.7. Let $x \in S-\Sigma$. If $x$ is not a complex point, then $m(x)=0$. If $x$ is a positive elliptic or negative hyperbolic point, then $m(x)=+1$. If $x$ is a negative elliptic or positive hyperbolic point, then $m(x)=-1$.

Next Lemma is used to show Theorem 1.(2).

LEMMA 1.8. The homology class $\left[\tilde{C}_{+} \cup\left(-\tilde{C}_{-}\right)\right] \in H_{4}(\tilde{G}, \mathbb{Z})$ is the Pioncaré dual of $\pi^{*} c_{1}(E) \in H^{2}(\tilde{G}, \mathbb{Z})$.

Proof: Consider the complex line bundle $\pi^{*}(E \wedge E)$ over $\tilde{G}$. Then $c_{1}\left(\pi^{*}(E \wedge E)\right)=$ $c_{1}\left(\pi^{*} E\right)$, (see $\left.[\mathrm{H}]\right)$. Taking a metric of $E$ compatible with $J$, we define the section $s$ of $\pi^{*}(E \wedge E)$ over $\tilde{G}$ by $s(\alpha)=v \wedge w$, where $\alpha \in \tilde{G}$ and $v, w$ are orthonormal basis of $\alpha$ compatible with the orientation of $\alpha$. If, in above, $e_{1}, e_{2}, e_{3}, e_{4}$ are orthonormal, then locally $s$ is represented by $s=\left(-x_{3}+\sqrt{-1} x_{2}\right) e_{1} \wedge e_{3}$. Therefore we see that the zero locus of $s$ with the induced orientation is equal to $\tilde{C}_{+} \cup\left(-\tilde{C}_{-}\right)$. This shows the required result.

To end this section, we prove the following: 
LEMMA 1.9. If $x$ is an elliptic (resp. hyperbolic) point, then $i(x)=0$ (resp. -2).

ProOF: First we follow the arguments in $[4, \S 4.1]$. Let $x$ be a transverse complex point. Then, by [5], [19], there exist coordinates $(u, v): S, x \rightarrow \mathbf{R}^{2}, 0$ and $\left(p_{1}, q_{1} ; p_{2}, q_{2}\right)$ : $M, f(x) \rightarrow \mathbb{C}^{2}, 0$ such that

$$
f(u, v)=\left(u, v,(1+2 \gamma) u^{2}+(1-2 \gamma) v^{2}+\phi(u, v), \psi(u, v)\right)
$$

with $\gamma \in \mathrm{R}, 0<\gamma \neq \frac{1}{2}$, ord $\phi \geq 3$, ord $\operatorname{ord}_{0} \psi 3$, and $J=J_{0}+$ higher order terms, for the standard complex structure $J_{0}$ on $\mathbb{C}^{2}$. If $0<\gamma<\frac{1}{2}$ (resp. $\gamma>\frac{1}{2}$ ), then $x$ is elliptic (resp. hyperbolic).

To compute $i(x)$, we take the Euler field $E=u(\partial / \partial u)+v(\partial / \partial v)$. Then

$$
J\left(f_{*} E\right)=\left(-v+P_{1}\right) \frac{\partial}{\partial p_{1}}+\left(u+Q_{1}\right) \frac{\partial}{\partial q_{1}}+P_{2} \frac{\partial}{\partial p_{2}}+\left(2\left((1+2 \gamma) u^{2}+(1-2 \gamma) v^{2}\right)+Q_{2}\right) \frac{\partial}{\partial q_{2}}
$$

with $\operatorname{ord}_{0} P_{1} \geq 2, \operatorname{ord}_{0} Q_{1} \geq 2, \operatorname{ord}_{0} P_{2} \geq 3, \operatorname{ord}_{0} Q_{2} \geq 3$. We set, for sufficiently small $\epsilon$, $0<\epsilon^{2}<\left|1-4 \gamma^{2}\right|$,

$f_{\epsilon}(u, v)=\left(u-\epsilon v+A, v+\epsilon u+B,(1+2 \gamma) u^{2}+(1-2 \gamma) v^{2}+C, 2 \epsilon\left((1+2 \gamma) u^{2}+(1-2 \gamma) v^{2}\right)+D\right)$

where $A=\epsilon P_{1}, B=\epsilon Q_{1}, C=\phi+\epsilon P_{2}, D=\psi+\epsilon Q_{2}$. Consider the map-germ $F: \mathrm{R}^{4}, 0 \rightarrow$ $\mathrm{R}^{4}, 0$ defined by $F\left(u, v, u^{\prime}, v^{\prime}\right)=f_{\epsilon}(u, v)-f\left(u^{\prime}, v^{\prime}\right)$.

Let $E_{4}$ denote the $\mathrm{R}$-algebra of function-germs on $\mathbb{R}^{4}, 0$ and $m$ the unique maximal ideal of $E_{4}$. For the ideal $I(F) \subset E_{4}$ generated by the components of $F$, we easily see that $m^{3} \subset I(F)+m^{4}$, therefore $m^{3} \subset I(F)$ by Nakayama's lemma. Hence $F$ is a finite map-germ and we see $i(x)=\operatorname{deg}_{0} F$.

Following [7], we calculate $\operatorname{deg}_{0} F$. The algebra $Q(F)=E_{4} / I(F)$ is generated by $1 ; u, v$ and $u^{2}$ over $\mathrm{R}$. The class $s$ of Jacobian of $F$ is equal to $-64 \epsilon^{2} \gamma\left(1+2 \gamma+\frac{\epsilon^{2}}{1-2 \gamma}\right) u^{2}$ in $Q(F)$. Define the functional $\varphi: Q(F) \rightarrow \mathrm{R}$ by $\varphi\left(u^{2}\right)=-1, \varphi(1)=\varphi(u)=\varphi(v)=0$. Then we see $\varphi(s)>0$ and the matrix of the bilinear form $\langle,\rangle_{\varphi}: Q(F) \times Q(F) \rightarrow \mathrm{R},\langle a, b\rangle_{\varphi}=\varphi(a b)$, is equal to

$$
\left(\begin{array}{cccc}
1 & 0 & 0 & -1 \\
0 & -1 & 0 & 0 \\
0 & 0 & \frac{1+2 \gamma}{1-2 \gamma} & 0 \\
-1 & 0 & 0 & 0
\end{array}\right)
$$

If $0<\gamma<\frac{1}{2}$ (resp. $\gamma>\frac{1}{2}$ ), then we have $\operatorname{deg}_{0} F=$ signature of $\langle,\rangle_{\varphi}=0$ (resp. -2 ). 


\section{Perturbations.}

Proof of Theorem 2: (1) As in $\S 0$, we denote by $e_{+}, e_{-}, h_{+}, h_{-}$the numbers of positive elliptic, negative elliptic, positive hyperbolic, negative hyperbolic complex points of $\tilde{f}$ respectively. Further denote by $\delta_{+}, \delta_{-}$the numbers of self-intersection points of index $+1,-1$ respectively. Then $d_{+}=e_{+}-h_{+}, d_{-}=e_{-}-h_{-}$and $\delta=\delta_{+}-\delta_{-}$.

We may assume that the self-intersections do not occur on the complex points. We set $W=\tilde{f}\left(D^{2}\right)$. We take a tangent vector field $v$ to $W$ along $\tilde{f}$ such that $v$ are directed outward on $\partial W$, near all complex points and all self-intersection points. We perturb $\tilde{f}$ to $f^{\prime}$ along the direction of $J v$. Set $W^{\prime}=f^{\prime}\left(D^{2}\right)$. Then $i(f)$ is equal to the sum of intersection indices of $W$ and $W^{\prime}$, which is equal to $\sum i(y)-\chi\left(W_{0}\right)$, where the sum runs over all complex points and self-intersection points, and $W_{0}$ means $W$ minus small balls centered at complex points and self-intersection points. Then $\chi\left(W_{0}\right)=1-\left(e_{+}+e_{-}\right)-\left(h_{+}+h_{-}\right)-2\left(\delta_{+}+\delta_{-}\right)$. By Lemmas 1.3 and 1.9, we have $\sum i(y)=-2\left(h_{+}+h_{-}\right)-4 \delta_{-}$. Hence,

$$
i(f)=e_{+}-h_{+}+e_{-}-h_{-}-1+2\left(\delta_{+}-\delta_{-}\right)=d_{+}+d_{-}-1+2 \delta .
$$

(2) The Maslov index $m(f)$ is equal to $\sum m(x)$, the sum running over all complex points of $\tilde{f}$. Then by Lemma $1.7, m(f)=e_{+}-e_{-}-h_{+}+h_{-}=d_{+}-d_{-}$.

Q.E.D.

Now we apply Theorem 2 to calculate $i$ and $m$ for the open Whitney umbrella using concrete perturbations.

EXAMPLE 2.1: We perturb the local model $f: \mathbf{R}^{2}, 0 \rightarrow \mathbf{R}^{4}, 0$ of the open Whitney umbrella into $f_{\epsilon}: \mathbf{R}^{2}, 0 \rightarrow \mathrm{R}^{4}, 0$ defined by $f_{\epsilon}(u, v)=\left(\frac{v^{3}}{3}, u, u v, \frac{v^{2}}{2}+\epsilon v\right)$, for sufficiently small $\epsilon>0$. Then we have $\delta=0, h_{-}=1$ and $e_{+}=e_{-}=h_{+}=0$. By Theorem 2, we see $i(f)=-2$ and $m(f)=1$. For the map-germ $f^{\prime}$ defined by $f^{\prime}(u, v)=f(u, v)$, we see $i(f)=-2$ and $m(f)=-1$.

For another perturbation $f_{\epsilon}$ of $f$, for instance, $f_{\epsilon}(u, v)=\left(\frac{v^{3}}{3}+\epsilon v, u, u v, \frac{v^{2}}{2}\right)$, we have $\delta=0, h_{-}=1, e_{+}=e_{-}=h_{+}=0$, when $\epsilon>0$, and $\delta=-1, e_{+}=1, e_{-}=h_{+}=h_{-}=0$, when $\epsilon<0$.

Combined with Lemmas 1.3, 1.7, 1.9, Remark 1.4 and Example 2.1, we get Table 3. 


\section{Implications.}

First we deduce the formulae of Lai and Givental' from Theorem 1.

ThE FORMula of LAI: By Table 3 , we have $\sum_{y}\{i(y)+1\}=d_{+}+d_{-}+\delta_{+}-3 \delta_{-}$, $V \cdot V=\nu+2\left(\delta_{+}-\delta_{-}\right), \chi(V)=\chi(S)-\left(\delta_{+}+\delta_{-}\right), \sum_{x} m(x)=d_{+}-d_{-}$, where $\delta_{+}$and $\delta_{-}$ are similar numbers as in the proof of Theorem 2. Thus $\chi(V)+V \cdot V=\nu+\chi+\delta_{+}-3 \delta_{-}$. By Theorem 1, we have $d_{+}+d_{-}=\nu+\chi$ and $d_{+}-d_{-}=c$.

The formula of Givental': By Table 3 , we have

$$
\sum_{y \in V}\{i(y)+1\}=\delta_{+}-3 \delta_{-}-T, \quad \chi(V)+V \cdot V=\chi-\left(\delta_{+}+\delta_{-}\right)+V \cdot V
$$

By Theorem 1.(1), we have $-V \cdot V=\chi-2 \delta+T$.

Proof of Theorem 1: (1) Denote by $X$ the set of singular points of $V$. We remove from $V$ small balls centered at points of $X$. Denote by $V^{\prime}$ the resulting surface with boundary. Let $v$ be a vector field over $V^{\prime}$ directed inward (relatively to $V^{\prime}$ ) along $\partial V^{\prime}$. Using $J v$, we perturb $V$. Then we see

$$
V \cdot V=\sum_{b \in X} i(y)-\chi\left(V^{\prime}\right), \quad \chi\left(V^{\prime}\right)=\chi(V)-\sharp X
$$

Thus we have $V \cdot V=\sum_{y \in X}\{i(y)+1\}-\chi(V)$.

(2) For $x \in \Sigma$, take a small disk $D_{x} \subset S$ around $x$. We extend the Gauss map $g$ : $S-\bigcup_{x \in \Sigma} D_{x} \rightarrow \tilde{G}$ to a section $\tilde{g}: S \rightarrow \tilde{G}$. Then the sum $\sum_{x \in S} m(x)$ is equal to the intersection number of $\tilde{g}(S)$ and $\tilde{C}_{+} \cup\left(-\tilde{C}_{-}\right)$. By Lemma 1.8, this number is equal to

$$
\left\langle\pi^{*} c_{1}(E), \tilde{g}_{*}[S]\right\rangle=\left\langle\tilde{g}^{*} \pi^{*} c_{1}(E),[S]\right\rangle=\left\langle c_{1}(E),[S]\right\rangle=c .
$$

Q.E.D.

\section{REFERENCES}

[1] Arnol'd, V.I., "Singularities of Caustics and Wave Fronts," Kluwer Academic Publishers, 1990.

[2] Banchoff, T., Farris, F., Tangential and normal Euler numbers, complex points, and singularities of projections for oriented surfaces in 4-space, Abstracts of Papers Presented to the Amer. Math. Soc. 13-1 (1992), p. 100.

[3] Bennequin, B., Entrelacements et équations de Pfaff, Astérisque 107-108 (1983), 87-161.

[4] Topologie symplectique, convexité holomorphe et structure de contact, Astérisque 189-190 (1990), 285-323.

[5] Bishop, E., Differentiable manifolds in complex Euclidean space, Duke Math. J. 32 (1965), 1-21.

[6] Chern, S.S., Spanier, E., A theorem on orientable surfaces in four-dimensional space, Comment. Math. Helv. 25 (1951), 205-209.

[7] Eisenbud, D., Levine, H.I., An algebraic formula for the degree of a $C^{\infty}$ map germ, Ann. of Math. 106 (1977), 19-44. 
[8] Fiedler, T., Exceptional points on surfaces in four-spaces, Ann. Global Anal. Geom. 3-2 (1985), 219-231.

[9] - A characteristic class for totally real surfaces in the Grassmannian of two-planes in four-space, Ann. Global Anal. Geom. 4-1 (1986), 121-132.

[10] Complex plane curves in the ball, Invent. Math. 95 (1989), 479-506.

[11] Friedrich, Th., On surfaces in four-spaces, Ann. Global Anal. Geom. 2-3 (1984), 257-287.

[12] Fukuda, T., Local topological properties of differentiable mappings, I, Invent. Math. 65 (1981/82), 227-250.

[13] Givental', A.B., Lagrangian imbeddings of surfaces and unfolded Whitney umbrella, Funkt. Anal. Prilozhen 20-3 (1986), 35-41.

[14] Singular Lagrangian varieties and their Lagrangian mappings, in "Itogi Nauki Tekh., Ser. Sovrem. Probl. Mat. (Contemporary Problems of Mathematics) 33," VITINI, 1988, pp. $55-112$.

[15] Hirzebruch, H., "Topological Methods in Algebraic Geometry," Springer-Verlag, 1956.

[16] Ishikawa, G., The local model of an isotropic map-germ arising from one dimensional symplectic reduction, Math. Proc. Camb. Phil. Soc. 111-1 (1992), 103-112.

[17] Lai, H.-F., Characteristic classes of real manifolds immersed in complex manifolds, Trans. Amer. Math. Soc. 172 (1972), 1-33.

[18] McDuff, D., The local behavior of holomorphic curves in almost complex 4-manifolds, J. Diff. Geom. 34 (1991), 143-164.

[19] Moser, J., Webster, S., Normal forms for real surfaces in $\mathrm{C}^{2}$ near complex tangents and hyperbolic surface transformations, Acta. Math. 150 (1983), 255-296.

[20] Ohmoto, T., Thom polynomials for isotropic mappings, Preprint.

[21] Viro, O.Y., Some integral calculus based on Euler characteristics, in "Lecture Notes in Math. vol. 1346," Springer, pp. 127-138.

[22] Weinstein, A., "Lectures on symplectic manifolds," Regional Conference Series in Math. 29, Amer. Math. Soc., 1977. 